\title{
Phoniatricians and otorhinolaryngologists approaching oropharyngeal dysphagia: an update on FEES
}

\author{
Antonio Schindler ${ }^{1}$ D $\cdot$ Laura W. J. Baijens $^{2,3}$ (D) $\cdot$ Ahmed Geneid $^{4}$ (D) $\cdot$ Nicole Pizzorni $^{1}$ (D)
}

Received: 18 May 2021 / Accepted: 26 October 2021 / Published online: 15 November 2021

(c) The Author(s), under exclusive licence to Springer-Verlag GmbH Germany, part of Springer Nature 2021

\begin{abstract}
Purpose Oropharyngeal dysphagia (OD) is a common phenomenon in otorhinolaryngology and phoniatrics. As both subdisciplines have a strong tradition and clinical experience in endoscopic assessment of the upper aerodigestive tract, the implementation of fiberoptic endoscopic evaluation of swallowing (FEES) was an almost self-evident evolution. This review aims to provide an update on FEES and the role of phoniatricians and otorhinolaryngologists using FEES in Europe.

Methods A narrative review of the literature was performed by experts in the field of FEES both in the clinical context and in the field of scientific research.

Results FEES is the first-choice OD assessment technique for both phoniatricians and otorhinolaryngologists. FEES is becoming increasingly popular because of its usefulness, safety, low costs, wide applicability, and feasibility in different clinical settings. FEES can be performed by health professionals of varying disciplines, once adequate knowledge and skills are acquired. FEES aims to determine OD nature and severity and can provide diagnostic information regarding the underlying etiology. The direct effect of therapeutic interventions can be evaluated using FEES, contributing to design the OD management plan. Standardization of FEES protocols and metrics is still lacking. Technological innovation regarding image resolution, frame rate frequency, endoscopic light source specifications, and endoscopic rotation range has contributed to an increased diagnostic accuracy.

Conclusion The rising number of phoniatricians and otorhinolaryngologists performing FEES contributes to the early detection and treatment of OD in an aging European population. Nevertheless, a multidisciplinary approach together with other disciplines is crucial for the success of OD management.
\end{abstract}

Keywords Deglutition · Deglutition disorders · Fiberoptic endoscopic evaluation of swallowing · Phoniatrics · Otorhinolaryngology

Antonio Schindler

antonio.schindler@unimi.it

1 Department of Biomedical and Clinical Sciences "L. Sacco", Milan University, UOS Foniatria, ASST FatebenefratelliSacco, Ospedale Sacco, Via GB Grassi 74, 20154 Milano, Italy

2 Department of Otorhinolaryngology, Head and Neck Surgery, Maastricht University Medical Center, Maastricht, The Netherlands

3 School for Oncology and Developmental Biology-GROW, Maastricht University, Maastricht, The Netherlands

4 Department of Otorhinolaryngology and Phoniatrics-Head and Neck Surgery, University of Helsinki and Helsinki University Hospital, Helsinki, Finland

\section{Introduction}

In the late 1980s, Dr. Langmore was the first to introduce a comprehensive swallowing study protocol using flexible endoscopy and named it fiberoptic endoscopic evaluation of swallowing (FEES) [1]. Today, FEES is considered one of the two gold-standard methods for the diagnosis of oropharyngeal dysphagia (OD), together with the videofluoroscopic swallowing study (VFSS) [2]. Advantages of FEES include absence of radiation exposure, being portable, and thus, feasible at the bedside for patients with limited mobility, and enabling visualization of non-radio-opaque secretions such as pharyngeal saliva residue. Despite the fact that FEES was initially developed by a speech and language pathologist (SLP), it is nowadays carried out by health professionals of various disciplines based on their curriculum 
(knowledge and skills) and on country-specific regulations. In many European countries, FEES is registered as a medical procedure and is usually performed by phoniatricians and otorhinolaryngologists.

The present narrative review aims to provide an update on FEES and the role of phoniatricians and otorhinolaryngologists using FEES in Europe.

\section{Phoniatricians, otorhinolaryngologists, and oropharyngeal dysphagia}

Phoniatrics and otorhinolaryngology are two different medical sub-disciplines involved in the management of upper aerodigestive tract disorders. While both sub-disciplines are closely related and exist since the beginning of the XX century, they have a different focus of attention. Otorhinolaryngology is devoted to the medical and surgical treatment of diseases of the oral cavity, pharynx, larynx, and proximal cervical esophagus; phoniatrics is focused on the recovery or maintenance of the voice, speech, language, hearing, and swallowing function where expertise in pre-habilitation and rehabilitation plays an important role [3, 4] (Union of the European Phoniatrics, https://www.uep.phoniatrics.eu/ downloads/logbook-phoniatrics_uems_update2010.pdf). Due to a shared common ground, it is, therefore, obvious that both sub-disciplines strongly rely on endoscopic procedures for the assessment of upper aerodigestive tract disorders and their management [5-8]. After the first description of in vivo assessment of the larynx by the singer and teacher Manuel Garcia in 1855 [9], experts in otorhinolaryngology and phoniatrics significantly contributed to the development of endoscopic techniques $[10,11]$, and immediately took advantage of the introduction of flexible endoscopes for the assessment of the upper aerodigestive tract [12, 13], first described in 1968 [14]. After the first report of fiberscope application for swallowing assessment by the SLP Susan Langmore in 1988 [1], phoniatricians and otorhinolaryngologists used this new technique for their patients' OD management and further refined it with the help of various technological innovations [15-18].

OD is a common phenomenon in everyday clinical practice of both otorhinolaryngologists and phoniatricians. In some cases, it is the first symptom of a still undiagnosed underlying disease leading the patients to seek for medical consultation, such as in the case of hypopharyngeal or proximal esophageal carcinoma, a Zenker diverticulum or a cervical osteophyte. In the majority of the referred patients, the underlying etiology of OD is known, such as the consequences of head and neck cancer treatment and multimodality treatment in particular, neurological disorders (e.g., traumatic brain injury, Parkinson disease, stroke, myotonic dystrophy) or congenital syndromic and non-syndromic diseases. In case of underlying cross-disciplinary diagnoses, it is important to manage OD in collaboration with physicians and allied-health professionals of these different disciplines as the treatment of the underlying condition may in some cases contribute to an improvement of OD. Think of conditions such as Parkinson disease and the use of levodopa or myasthenia gravis. Depending on the tradition and history of otorhinolaryngology and phoniatrics which may vary according to different regions and countries in the world as well as the personal preferences and interests of the physician, the following patient populations are managed: head and neck cancer patients, frail elderly patients, adults or children with neurological disorders, critically ill patients following tracheotomy or tracheal intubation, children with upper airway malformations, and patients with OD of unknown origin. [19-21]. Because of the long history of endoscopic assessment in everyday clinical practice for both otorhinolaryngologists and phoniatricians, FEES represents the first choice for OD assessment [22, 23]. For the optimal integration of FEES findings in the OD management plan, a multidisciplinary collaboration with other disciplines (nurses, dieticians, occupational therapists, SLPs, intensive care specialists, pediatricians, neurologists, pulmonologists, geriatricians, doctors in physical medicine and rehabilitation) involved in the care of a patient is indispensable [24, 25].

\section{Increasing popularity of FEES}

After its initial introduction in the USA, the FEES technique was introduced and implemented fairly quickly in Europe and the rest of the world, becoming increasingly popular. Several factors contributed to its popularity, including its patient safety, low costs, wide applicability and in particular its usefulness in daily clinical practice. FEES is a safe procedure across the age spectrum; its potential adverse events include epistaxis, vasovagal syncope and laryngospasm, each occurring in less than $2 \%$ of the patients [20, 26-30]. In several studies on thousands of patients, complications were all self-limiting, resolved without any sequelae, and showed no correlation with the endoscopists' track record. For these reasons, FEES is often performed without the application of lubricants or topical anesthetics. It has also been described that the application of $0.2 \mathrm{ml}$ lidocaine in the nasal cavity makes the examination more convenient and tolerable for patients without affecting the FEES outcome in terms of an iatrogenic disruption of sensory function of the pharynx and larynx [31].

As far as we know no studies have been published on the cost-benefit analysis of FEES, but it is well known that the basic instrumentation to perform a FEES (light source and endoscope) is affordable and that even more advanced 
endoscopic equipment, including a video-endoscope, processor, narrow-band imaging (NBI), and recording system, is significantly cheaper than the other gold-standard tool for swallowing assessment, namely an X-ray machine for a VFSS. These relatively lower purchase costs make the FEES examination accessible to health professionals with a limited budget and to patients in countries with a lower mean-citizen's income. This has important implications, as it further contributes to the implementation of FEES across and within countries providing the opportunity to serve the underserved currently being the most important issue in OD management all over the world. Usually countries with a lower mean-citizen's income do not have FEES trained or certified clinicians, any health professionals with expertise in OD management, or skilled technicians to maintain and repair the instruments. This may be a bottle neck for the implementation of FEES.

Another factor that makes FEES popular is its applicability as a bedside examination in different settings including intensive care units (ICU), neonatal ICU (NICU), nursing homes, and even patients' home, further improving the accessibility of swallowing assessment for various patient populations [32-34]. In addition, performing FEES at the beside with adequate personal protective equipment [35] and/or using single-use endoscopes, prevents hospitalized patients from having to be transported to an X-ray machine for a VFSS with the risk of spreading or becoming infected with dangerous microorganisms such as multidrug-resistant bacteria (e.g., Acinetobacter baumannii, New Delhi metallo$\beta$-lactamase-producing Klebsiella pneumoniae) or viruses such as the SARS-CoV-2. High-level disinfection preferably using an automatic endoscope reprocessor is recommended as the minimum level of disinfection required for reprocessing flexible nasopharyngoscopes. It is important to adhere to the clinical practice guidelines for endoscope reprocessing to ensure patient safety, and in many countries, this is a legal requirement $[36,37]$. The factor that probably contributed most to the popularity of FEES is its usefulness and accessibility in daily clinical practice, as FEES significantly contributes to the clinical understanding of the OD phenotype and subsequently to OD management allowing among others' dietary recommendations [38-42].

\section{Who are the health professionals carrying out FEES?}

The FEES exam was initially described by an SLP and subsequently developed and refined by among others otorhinolaryngologists, phoniatricians, and neurologists in collaboration with engineers, and manufacturers. Nowadays, FEES is carried out by health professionals of various disciplines as described above. The exact nature of the discipline of the health professional seems to play a less important role than the FEES examiner's level of expertise in OD and adequate knowledge and skills to carry out and interpret a FEES examination (Table 1) [22, 23, 43-46]. Patients are being 'put at risk' when health professionals are performing FEES without proper training or supervision. Incomplete or incorrect information will end up in recommendations with a higher risk of OD management-related adverse events.

Knowledge and skills to perform FEES are sometimes part of the standard training curriculum of a particular profession, as in the case of phoniatricians. Otherwise, a

Table 1 Recommended knowledge and skills to carry out a FEES examination [21, 22, 42-45]

\begin{tabular}{|c|c|}
\hline Knowledge & Skills \\
\hline $\begin{array}{l}\text { Respiratory and swallowing physiology as well as airway protection } \\
\text { mechanisms across lifespan (from infancy to adulthood and high age) }\end{array}$ & $\begin{array}{l}\text { Handle a flexible endoscope avoiding instrument damage or spreading } \\
\text { of microorganisms (contamination) }\end{array}$ \\
\hline $\begin{array}{l}\text { Endoscopic anatomy of the pharynx and larynx across lifespan (from } \\
\text { infancy to adulthood and high age) }\end{array}$ & $\begin{array}{l}\text { Handle topical anesthetics, when necessary or indicated } \\
\text { Perform endoscopy without or minimal patient discomfort }\end{array}$ \\
\hline $\begin{array}{l}\text { Anatomical and physiological impairments affecting swallowing across } \\
\text { lifespan (from infancy to adulthood and high age) }\end{array}$ & $\begin{array}{l}\text { Avoid and manage common adverse events (nose bleeding, syncope, } \\
\text { etc.) }\end{array}$ \\
\hline \multirow{2}{*}{$\begin{array}{l}\text { Indication and contraindication of a FEES examination } \\
\text { Dysphagia treatment modalities (medical, surgical, (p)rehabilitative, } \\
\text { etc.) }\end{array}$} & $\begin{array}{l}\text { Handle the endoscope to get the required view of the upper aerodiges- } \\
\text { tive tract }\end{array}$ \\
\hline & Make the patient perform tasks and maneuvers to achieve a complete \\
\hline \multicolumn{2}{|l|}{$\begin{array}{l}\text { Indication for additional diagnostic assessment procedures if necessary } \\
\text { (CT scan, HRM, etc.) }\end{array}$} \\
\hline \multicolumn{2}{|l|}{ Timing and frequency of FEES follow-up } \\
\hline \multirow{2}{*}{$\begin{array}{l}\text { Use FEES outcome to counsel patients, care-givers, and health profes- } \\
\text { sionals of the team }\end{array}$} & Interpret the examination and release a written report \\
\hline & $\begin{array}{l}\text { Design a dysphagia management plan based on the findings of the } \\
\text { FEES examination, the underlying disease, and according to the } \\
\text { setting of care }\end{array}$ \\
\hline
\end{tabular}

$C T$ computed tomography, HRM high-resolution manometry, FEES fiberoptic endoscopic evaluation of swallowing 
certified training curriculum to carry out a FEES examination is recommended [47]. The regulations on FEES training and certification usually vary by country. An example of such a FEES curriculum for neurogenic and geriatric OD has been developed by the European Society for Swallowing Disorders (ESSD) [47]. Key elements in this curriculum for the development of knowledge and skills to perform FEES are: (1) workshops to acquire knowledge on OD, FEES protocols, FEES metrics, and to develop skills to handle and pass the endoscope on a medical dummy; (2) a FEES clinical internship to carry out FEES examinations on patients with direct and indirect supervision by a certified and trained FEES instructor. A similar program has been recently developed for nurses in Japan [48].

In addition, specific national legal provisions define which professions are allowed to perform FEES. For instance, endoscopy is characterized as a medical act in some countries, and as such, must be performed by medical doctors.

Regardless of the professional performing FEES, it is advisable that both the ENT or the Phoniatrician and the SLP attend the FEES examination. A team approach yields several advantages. The combination of the medical expertise in the diagnosis and the SLP's expertise in the functional assessment and the treatment eases the decision making on OD treatment broadens the scope on the swallowing impairment and the patient's need and promotes a continuum in the patient management from the diagnosis to the treatment. In addition, an interdisciplinary approach provides a gain in the degree of agreement in FEES interpretation. As literature suggests, the discussion among clinicians when scoring FEES findings improves concordance compared to independent rating of FEES exams [49].

\section{The aim of a FEES examination}

FEES can be applied in both clinical practice and scientific research. In clinical practice, FEES enables the management of patients who complain of or who suffer from OD. It has two main aims: (1) to contribute to the diagnosis of the underlying disease causing OD, if unknown; (2) to prescribe a patient-tailored OD treatment plan (medical, surgical, and rehabilitative, including compensatory, behavioral or neuromodulation approaches). In some patients, OD can be the initial presenting symptom of an undiagnosed underlying disease, such as Zenker diverticulum, Parkinson disease, head and neck cancer, amyotrophic lateral sclerosis, and myasthenia gravis. In all the cases with an undiagnosed underlying disease, detailed information on the anatomical, sensory-motor findings, and effects of compensatory strategies must be obtained and discussed with the physician or medical team in charge of the patient. In some cases, this information is pathognomonic for the underlying disease, such as the sign of the rising tide in case of a Zenker diverticulum [50], while in other cases, additional clinical information is necessary to determine the underlying diagnosis, such as a vocal fold movement disorder in multiple system atrophy [51, 52].

In patients with OD of known underlying etiology, FEES should provide information to: (1) verify that the swallowing pathophysiology or OD phenotype is 'valid' for the underlying diseases (e.g., tongue pumping movements and cueing effects are findings that may be consistent with Parkinson disease [53]); (2) select the optimal dietary conditions (modified texture diets and level of thickened liquids); (3) design a patient-tailored OD treatment plan (pharmacological, surgical, and rehabilitative, or combinations hereof), based on the underlying etiology and swallowing pathophysiology; (4) verify treatment outcomes and disease progression over time [54-56].

In scientific research, FEES can be used to score OD severity, determine OD phenotype, and assess the effectiveness of OD treatment. However, concerns about the quality of psychometric properties of visuoperceptual measures are described in the section "FEES scoring and interpretation" [57].

\section{What are the indications and who are the patients undergoing FEES?}

FEES can be performed across the age spectrum, in almost any clinical setting and various pathophysiological conditions; nonetheless, there are cases in which FEES is relatively or absolutely contraindicated or of limited value [58]. FEES is contraindicated in patients with a respiratory rate $>35 / \mathrm{min}$, in those who have an impaired consciousness (coma, vegetative state, minimally conscious state), and in patients who refuse any form or attempt of oral food administration. In patients with a high respiratory rate, the swallowing-breathing coordination is challenging as the swallowing apnea is shortened. These circumstances may promote aspiration and further increase breathing impairment [59].

In patients with complete oral food refusal, swallowing anatomy including the integrity of the cranial nerves and saliva management can be explored during endoscopy providing only a part of the information necessary for clinical decision making on OD treatment. Without the administration of oral bolus, the pharyngeal and laryngeal sensorymotor function and the effectiveness of compensatory strategies (postural maneuvers, bolus modification, and maneuvers) cannot be assessed reliably. Therefore, information on swallowing physiology in particular swallowing safety and efficiency cannot be determined for these patients. 
In some of these patients, no abnormalities are seen during saliva swallowing while others have abnormalities that explain the refusal of oral foods such as painful oropharyngeal ulcers. The FEES examination may also be of limited value in patients who accept oral nutrition but are incapable of oral bolus transport such as after a stroke. In these cases, FEES can provide information on preswallow posterior spill as a result of impaired glossopalatal closure and the presence of an intact pharyngeal swallow which is of added value in treatment decision making.

Prior to performing a FEES examination, it is important to obtain the following information to guarantee careful, effective diagnostics and patient safety, namely: (1) the patient history including information on demographic characteristics (age, gender, height, weight, etc.), comorbid diseases and preferably the underlying OD etiology, medication, previous treatment for OD and pulmonary complications; (2) the complaints of the patient for whom he/she is seeking help, as the explanation of FEES findings to the patient and his/her care-giver must be aligned with this initial anamnestic information; (3) detailed description on the indication and purpose of the FEES examination for this particular patient (i.e., diagnosis of the underlying disease, clinical description of OD phenotype); (4) detailed information on diet and meal characteristics (including information on the setting/conditions of the mealtime, eating duration, patient's eating behavior, self-feeding skills, and aids such as adapted cutlery or a customized chair).

Other important patient-related aspects that should be known before the start of the FEES examination are the patient's level of consciousness, cognitive skills, compliance, laryngeal function (voice, cough), integrity of oral structures and oral motor function, dental status, and allergy status [60].

\section{FEES protocol}

There is currently no consensus on the content of a FEES protocol. In the literature, various FEES protocols are described that differ according to the nature of the clinical setting (e.g., stroke unit, neurological OD outpatient clinic, and otorhinolaryngological OD outpatient clinic), the underlying condition (i.e., stroke, Parkinson disease, myasthenia gravis, and critically ill neurological patients), the personal preference and habits of a health professional or the multidisciplinary team [54, 55, 61-63]. In the majority of previously described protocols, there is a clear preference for standardization of the preparation and execution of the FEES examination such as patient positioning, bolus consistency and volumes, number of swallows, and method of oral administration. However, there are also protocols that recommend a more 'natural' observation of the swallowing physiology, for example by letting the patient eat himself [64]. The question that immediately arises is whether it is possible at all to speak of a 'natural' eating process during a FEES examination that is a reflection of a meal at home? Eating with an endoscope in the upper aerodigestive tract, eating in very close proximity to a health professional (in the personal space), listening to instructions of a health professional, and eating outside of a familiar environment are all conditions that cause higher central neurological processes to change compared to eating in the familiar conditions at home (or the usual environment) [65]. Standardization of a FEES protocol offers the advantage that the test conditions before and after a treatment or over time during follow-up stay exactly the same and changes in the FEES outcome measurements are not caused by changes in the FEES protocol.

The FEES examination is usually performed using a standardized protocol, preferably with the patient in an upright position; however, the examination can be modified and customized if necessary, depending on patient's usual position during meals. During FEES, the patient faces either the monitor or the examiner. Currently, there is insufficient scientific evidence that visual biofeedback by looking at the monitor gives rise to beneficial effects on central neurological processes involved in swallowing [66, 67].

Observing the various existing FEES protocols, a number of similarities are visible regarding the mains steps to follow during a FEES examination, namely the evaluation of the swallowing endoscopic anatomy, pharyngeal and laryngeal sensory-motor function, saliva and bolus management, and the effectiveness of compensatory strategies $[15,64,65$, $68]$. Anatomical assessment should include a detailed and systematic exploration of the surface mucosa and the underlying structures of the nose, pharynx, and larynx. In some cases, specific maneuvers such as the trumpet or Valsalva maneuver are helpful to explore the hypopharynx and postcricoid region, or head turn maneuvers to explore the pyriform sinuses in case of cervical osteophytes [69]. In patients with a tracheostomy, the tracheobronchial tree should be explored following removal of the tracheostomy tube, if possible, to examine the subglottal structures by inserting the endoscope into the stoma and flexing it upwards [70, 71]. Motor assessment should be performed systematically including velopharyngeal, pharyngeal (squeeze maneuver) [72], tongue base, and laryngeal motor function at rest (spontaneous swallowing function and frequency), during speech and non-speech tasks including sustained contraction and rapid diadochokinetic movements. Subsequently, motor assessment should be integrated with sensory testing of the pharynx and larynx in the form of touching pharyngeal and laryngeal structures with the tip of the endoscope and analyzing patient's reaction. 
During anatomical and sensory-motor assessment, saliva residue severity rating is carried out as well. However, the core business of the FEES examination is to evaluate the swallowing function during the administration of various food and liquid consistencies and volumes. During this process, the health professional pays particular attention to the main signs of swallowing impairment such as spillage, penetration/aspiration, residue, and regurgitation and tries to 'translate' these findings to the underlying pathophysiology to obtain an integrated phenotypic OD description. Since no standard FEES protocol exists as to the number and rheological characteristics of the consistencies, their volumes, number of trials for each volume and consistency, large variations can be observed across and within countries. Nevertheless, there is general consensus that at least thin liquids, semisolids, and solid consistencies should be tested strictly monitoring patient safety. Food and liquids are usually dyed to improve judgment of airway invasion, although diverting opinions exist on this topic [73-75]. Recently, Curtis et al. described that airway invasion can be observed more frequently and with a higher level of reliability using barium compared to the use of blue- and green-dyed water during a FEES examination [76]. While the use of barium seems to increase diagnostic accuracy, there are potential problems with its applicability as the necessary X-ray machine is not always available in different clinical settings (e.g., rehabilitation unit and community nursing home).

There is no consensus as to the minimum number of trials for each volume and consistency, but less than three trials per volume and consistency are likely to underestimate the risk of aspiration. It has been shown that the likelihood of detecting aspiration increases with the number of swallow trials, especially with thin liquids in neurological patients, showing an increase up to the 9th swallow trial, while the likelihood of detecting aspiration with semisolids in oncological patients plateaus after four boluses [77]. In some patients, the first swallow trial may be considered as a 'warm-up' and the severity of pathophysiology may improve in subsequent swallows [78]. It is speculative to assume that this finding has no clinical relevance and is due to a learning curve of the patient. Maybe this happens at home as well every time liquids or foods are started. For example, in some neurological phenotypes, the pharynx can improve its sensory-motor activity after the initial swallow [79]. Finally, next to the core business of FEES 'evaluating the swallowing function' the therapeutic effect of compensatory strategies such as head postures, swallowing maneuvers, and bolus modification is also an important point of attention (Table 2) [80-82].

As there is general consensus on the main steps on how to carry out a FEES, there are a number of similarities in various existing FEES protocols that also indicate a consensus on the need for tailoring the examination to a single patient [78, 79, 83]. Firm scientific evidence on the effect of a patient-tailored FEES protocol compared to a standardized protocol is lacking. There are no randomized, blinded studies that, among other things, compare the design and outcome of different FEES protocols. Nevertheless, experts share the opinion that a patient-tailored FEES protocol based on information from multiple domains (including cognition,

Table 2 Swallowing postures and maneuvers feasible and of added value during a FEES examination [79-81]

\begin{tabular}{|c|c|c|}
\hline Impairment & Posture/maneuver & Rationale \\
\hline $\begin{array}{l}\text { Delayed initiation of the pharyngeal reflex and oral } \\
\text { incompetence (preswallow posterior spill) }\end{array}$ & Chin down & $\begin{array}{l}\text { Widens the valleculae and narrows the laryngeal vesti- } \\
\text { bule reducing the risk of aspiration }\end{array}$ \\
\hline Reduced posterior displacement of the tongue base & Chin down or effortful swallow & $\begin{array}{l}\text { Pushes the tongue base towards the posterior phar- } \\
\text { yngeal wall; increases the posterior tongue base } \\
\text { movement }\end{array}$ \\
\hline $\begin{array}{l}\text { Unilateral vocal fold paralysis or post-cordectomy or } \\
\text { delayed vocal fold closure }\end{array}$ & $\begin{array}{l}\text { Head rotation to the affected } \\
\text { side and/or supraglottic } \\
\text { swallow }\end{array}$ & $\begin{array}{l}\text { Places extrinsic pressure on the thyroid cartilage } \\
\text { improving vocal fold approximation and directing the } \\
\text { bolus via the unaffected side; voluntary breath hold } \\
\text { usually closes the vocal folds before and during the } \\
\text { swallow }\end{array}$ \\
\hline Unilateral oropharyngeal paralysis or weakness & Head tilt to the unaffected side & Directs the bolus via the unaffected side using gravity \\
\hline UES dysfunction & Head rotation & $\begin{array}{l}\text { Pulls cricoid cartilage away from the posterior pharyn- } \\
\text { geal wall reducing the resting pressure in the UES }\end{array}$ \\
\hline Incomplete closure of the airway entrance & Super-supraglottic swallow & $\begin{array}{l}\text { Effortful breath hold tilts the arytenoids forward, } \\
\text { closing the airway entrance before and during the } \\
\text { swallow }\end{array}$ \\
\hline $\begin{array}{l}\text { Reduced displacement and/or duration of hyolaryn- } \\
\text { geal elevation }\end{array}$ & Mendelsohn maneuver & Prolongs the UES opening \\
\hline $\begin{array}{l}\text { Reduced duration of tongue base retraction and swal- } \\
\text { low }\end{array}$ & Mendelsohn maneuver & $\begin{array}{l}\text { Prolongs the posterior movement of the tongue base to } \\
\text { the pharyngeal wall }\end{array}$ \\
\hline
\end{tabular}


dental status, pulmonary status) can be useful [83]. A patient-tailored FEES protocol can include the observation of the effect of self-feeding skills on the swallowing function, offering adjusted consistencies in patients without dentition or a clinically strong suspicion of severe aspiration, offering foods that are highly appreciated by patients with intellectual disabilities.

The main steps to follow during a FEES examination as described above can also be applied in the pediatric population [84-86] and even in breast-fed children [87]. Finally, disease-specific FEES protocols have been developed in recent years, representing a major step forward in tailoring the FEES examination to clinical needs $[54,55,61-63]$.

\section{FEES scoring and interpretation}

Currently, there is no consensus regarding a gold-standard measure, metrics or measurement protocol to analyze FEES video recordings [57]. Measures require sound psychometric properties to be suitable for clinical or scientific research purposes. A systematic review by Swan et al. assessed the quality of psychometric properties of visuoperceptual measures in FEES examinations using the Consensus-based Standards for the selection of health Measurement Instruments (COSMIN) framework. The authors concluded that there was insufficient evidence to recommend any individual measure included in their review as valid and reliable to interpret FEES examinations. Further research on this knowledge gap was recommended.

Despite the lack of methodologically high-quality FEES metrics, a standardized and clinically relevant measurement protocol for the interpretation of FEES examinations will have to be chosen in anticipation of the development of psychometrically robust measurements. A clinical assessment of the nature and severity of OD during FEES and appropriate treatment recommendations will have to be made. Anatomical and motor findings are mainly scored using categorical variables $[57,63,88]$.

Abnormal surface mucosa findings may include signs of infection, inflammation, ulcers, and tumors. Moreover, swelling or edema of the surface mucosa can be found and patients who underwent head and neck surgery may present with a significantly altered anatomy.

Abnormal anatomical findings may not only affect swallowing, but may also have potential implications for the patient's prognosis such as in case of recurrent head and neck cancer and must be interpreted with the support of an otorhinolaryngologist or phoniatrician.

Abnormal motor findings may include involuntary movement, and impaired movement. These impaired motor findings are usually related to the underlying disease or condition which may require the support of a neurologist especially in patients without a known diagnosis. During the assessment of motor findings, the squeeze maneuver (pharyngeal contraction induced by a high pitch, high pressed/i/ production) is clinically very relevant as it is a reliable and valid measure of pharyngeal motor integrity $[89,90]$ (see Fig. 1) and associated with the risk of penetration and aspiration [91].

The laryngeal sensory function is currently tested by touching the laryngeal structures with the tip of the endoscope and can be scored based on the absence or presence of one or more of the following findings: laryngeal adduction, swallowing, gagging, patient reporting the sensation of the laryngeal touch. Laryngeal sensory testing is reliable but requires adequate training of the health professional [92]. Although pressure applied to the larynx using the touch method is highly variable, indicating potential diagnostic inaccuracy in determining laryngeal sensory function [93], its clinical application still seems relevant as absence of laryngeal sensibility has been associated with the development of pneumonia [94, 95].

Commonly used FEES measures or scoring methods are based on findings such as preswallow pharyngeal spillage, penetration/aspiration, and pharyngeal residue.

Spillage, the passage of the head of the bolus into the pharynx prior to swallowing is usually the result of an impaired glossopalatal junction closure due to various etiologies. The scoring method of spillage various across studies and questions arise on its psychometric properties [49, $96,97]$. However, the observation of spillage during a FEES exam should lead to the search for improvement strategies usually on a patient-tailored basis such as the chin-down/ up maneuver [98, 99], cued swallowing [100], single versus

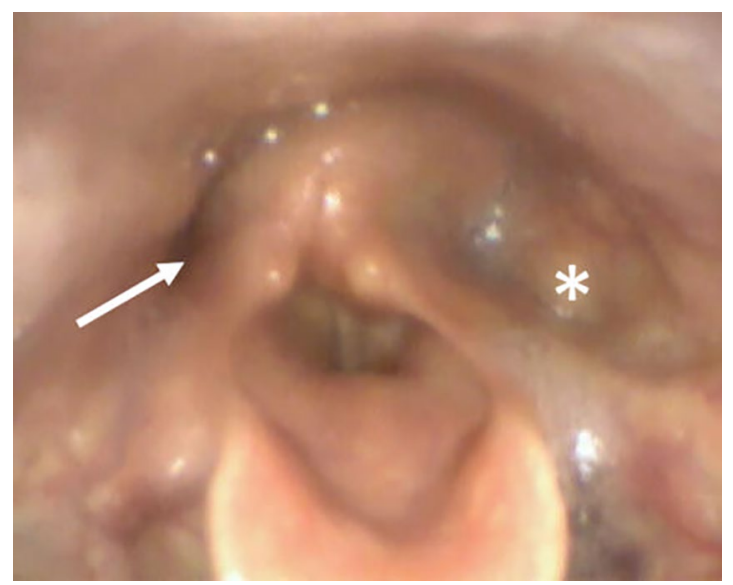

Fig. 1 An endoscopic view of the larynx and pharynx in a patient with a left pharyngeal wall paralysis during the squeeze maneuver. The absence of left pharyngeal wall movement without narrowing of the oro- and hypopharynx during contraction is shown by the white asterisk and is clearly asymmetrical compared to the contralateral side (white arrow) 
sequential swallowing [101], bolus volume or consistency modification [102-104], thermo-stimulation, and chemo or tactile-stimulation [105-107]. The so-called 'bolus holding test' can be helpful to distinguish spillage due to oral incompetence (preswallow or premature spillage) from a delayed initiation of the pharyngeal reflex [68].

The occurrence of penetration/aspiration during a FEES exam is an important finding in the context of swallowing safety. Penetration/aspiration is usually the result of an impaired upper airway closure due to various etiologies making its timing (preswallow, during swallowing, and postswallow) and amount of penetrated/aspirated bolus important addition findings in the translation towards a pathophysiological OD phenotype. If penetration/aspiration occurs before swallowing it will happen before the start of the whiteout (explained below) usually due to premature bolus spillage. Penetration/aspiration during the swallow so in the time frame of the whiteout is usually the result of an impaired upper airway closure and postswallow penetration/ aspiration may be the result of secondary leakage from pharyngeal residue or gastroesophageal regurgitation [108]. The scoring method of penetration/aspiration varies across studies and questions arise on its psychometric characteristics as well. The most well-known scoring system is the Penetration Aspiration Scale (PAS) by Rosenbek et al. [88]. Although originally developed for VFSS, the PAS has also been modified for the FEES examination [109, 110]. To increase the reliability and accuracy of penetration/aspiration scoring, it is recommended to record the examination on a privacyprotected network drive or hard disk and carefully analyze the recordings preferably using slow-motion and frameby-frame media player settings [111]. Recently, a modified FEES-derived PAS version was published for the assessment of penetration/aspiration in case of anatomical changes at the level of the larynx following surgery [112]. Shortcomings of the PAS are the lack of information about the estimated amount of bolus that was aspirated and the timing of the aspiration. In addition, variations in the interpretation or statistical processing of PAS outcomes have been described such as only reporting or using the worst PAS score of the entire FEES examination [113] or while other studies report and process separate PAS scores for each bolus trial [114, 115]but also different statistical approaches to PAS analyses are subject of debate [118].

The occurrence of pharyngeal residue during a FEES exam is an important finding in the context of swallowing efficiency. Pharyngeal residue is usually the result of an impaired ability to transfer the bolus from the mouth to the stomach due to various etiologies. Usually pharyngeal residue is scored by site (valleculae, pyriform sinuses) and amount of residue and variations between different measurement scales are based on variations in the number and description of the categorical levels of the scales (ordinal description of percentage of filling of the valleculae and pyriform sinuses, visual analog scale (VAS) scores of percentage of pooled bolus, anatomically based landmarks of pooling severity) and the timing of scoring (after the first swallow, after clearing swallows) [49, 116-119]. In addition, measurement scales to score saliva pooling (site, amount, and response to saliva pooling) have been developed [120, 121]. The most widely used scale for saliva residue severity rating by Murray et al. [120], scores the amount and "clearing' management of saliva residue. Recently, a more refined saliva residue severity scale was developed [121, 122] which scores residue site, amount, and response to saliva pooling and can predict the risk of developing aspiration pneumonia. Scales for pharyngeal residue severity rating may score residue site, amount, and 'clearing' management of pooled bolus [116-119]. The Yale Pharyngeal Residue Rating Scale (YPRRS) [118, 119], an anatomically defined and image-based tool, is a 5-point ordinal scale with a separate residue severity score for the valleculae and the pyriform sinuses. The major limitation of all these pharyngeal residue severity scales lies in the fact that their rating is carried out on preselected images of video frames, while FEES is capturing a dynamic swallowing process. Another limitation of the YPRRS is that it is unclear how to score unilateral residue compared to bilateral residue. The Boston Residue and Clearance Scale [117] is a very detailed, but rather complex scale consisting of an 11-point multidimensional scale, scoring amount of residue, location of residue in 4 different sites, and response to residue. Although this scale has shown excellent reliability, high concurrent validity, and internal consistency, a clear instruction manual how to apply this scale is missing. Furthermore, the scale was only published in the initial validation study which raises questions about its user-friendliness. The pooling score of Farneti [116] is obtained using separate ratings by pooling site, amount of residue, and response to residue. The main drawbacks of this scale are the lack of severity definitions and anatomical landmarks. Measuring visuoperceptual FEES variables remains a challenging task when it comes to the reproducibility of measurements [49]. The solution probably does not lie in the development of new scales with even more categories or VAS scores, since scoring a scale must also remain clinically feasible and a scale with more categories or VAS scores does not guarantee that the reproducibility of the measurement will improve [123, 124].

Scoring the severity of a safety or efficiency-related FEES variable is not a goal in itself and usually provides only 'indirect' information on the swallowing pathophysiology. FEES findings representing main signs of swallowing impairment need to be 'translated' to the underlying pathophysiology to obtain an integrated phenotypic OD description. This is an important step towards the design of an OD treatment plan (Table 3). 
Table 3 Main findings/signs of OD and their pathophysiological interpretations during a FEES examination $[48,64,67,107]$

\begin{tabular}{|c|c|}
\hline FEES sign/finding & Interpretation \\
\hline \multirow[t]{2}{*}{ Preswallow posterior spillage } & Impaired oral competence (positive bolus holding test) \\
\hline & Delayed initiation of the pharyngeal reflex (negative bolus holding test) \\
\hline \multirow[t]{2}{*}{ Vallecular residue } & Impaired tongue base retraction \\
\hline & Pharyngeal obstruction \\
\hline \multirow[t]{2}{*}{ Pyriform sinus residue } & Impaired pharyngeal contraction \\
\hline & Impaired hyolaryngeal elevation \\
\hline \multirow[t]{2}{*}{ Post-cricoid residue } & Impaired UES opening \\
\hline & Proximal esophageal obstruction \\
\hline \multirow[t]{2}{*}{ Rising tide sign } & Impaired UES opening \\
\hline & Zenker diverticulum \\
\hline \multirow[t]{2}{*}{ Delayed regurgitation } & Esophageal motor impairment \\
\hline & Zenker diverticulum \\
\hline Preswallow penetration/aspiration & Preswallow posterior spillage before airway closure/protection \\
\hline Per- or intraswallow penetration/aspiration & Impaired glottis closure (anatomical or functional impairment) \\
\hline Postswallow penetration/aspiration & $\begin{array}{l}\text { Vallecular residue and/or piriform/post-cricoid residue or regurgitation } \\
\text { with overflow into the laryngeal vestibule }\end{array}$ \\
\hline Reduced/absent whiteout & Poor tongue base retraction/pharyngeal contraction \\
\hline \multirow[t]{2}{*}{ Multiple swallows per bolus } & Fragmentation \\
\hline & Sequential swallowing (weakness, obstruction) \\
\hline \multirow[t]{2}{*}{ Nasal regurgitation } & Velopharyngeal insufficiency (anatomical or functional impairment) \\
\hline & Pharyngeal obstruction \\
\hline
\end{tabular}

UES upper esophageal sphincter

This translation of FEES findings is often based on assumptions because direct evidence such as EMG or highresolution manometry (HRM) measures at the same time are lacking. For example, vallecular residue is expected to increase with higher bolus viscosities and is usually interpreted as a result of a poor tongue base retraction, unless clinically relevant pharyngeal obstruction is present. Residue in the post-cricoid region is usually interpreted as an impairment resulting from upper esophageal sphincter (UES) dysfunction and/or proximal esophageal obstruction. In the case of UES impairment, an improvement would be expected with increasing bolus volumes [125]. Residue in the pyriform sinus is usually interpreted as the result of impaired pharyngeal contraction and/or hyolaryngeal elevation [126, 127]. This residue is expected to increase with higher viscosities and/or bolus volumes. An important FEES-specific swallowing variable is the so-called whiteout. During the swallow, a flash of white light related to the decreasing distance between the pharyngeal walls and the light from the endoscope (pharyngeal constrictor muscle contraction) prevents a direct view on the pharynx and larynx [128, 129]. An uncommon but important finding is the absence of a whiteout in case of severe impairment of the pharyngeal constrictor muscle contraction and tongue base retraction. Finally, the finding of multiple swallows per bolus trial is often called 'piecemeal deglutition'. The meaning of this concept is also open to interpretation and translation towards swallowing pathophysiology is also based on an assumption. It has been described as fragmentation of the bolus or sequential swallowing on the same bolus maybe serving as a compensation strategy to avoid penetration/aspiration [130], or due to impaired oropharyngeal bolus flow based on muscle weakness or pharyngeal obstruction. To the best of our knowledge, no validated scales exist for the pathophysiological interpretation of a FEES examination. Some detailed proposals have been published $[68,131]$, as reported in Table 4.

\section{The role of FEES in oropharyngeal dysphagia diagnostics}

To manage a patient with OD, the underlying disease should be diagnosed if possible and preferably explain or relate to the swallowing pathophysiology. It remains challenging when an OD phenotype is inconsistent with an underlying disorder. The question then is whether the underlying lesions or diseases responsible for the OD phenotype were fully revealed? Although FEES can assist in diagnosing various structural and motor disorders, additional diagnostic examinations such as a computed 
Table 4 Swallowing pathophysiology which can be inferred after a FEES examination according to Desuter [67] and Warnecke et al. [134]

\begin{tabular}{ll}
\hline $\begin{array}{l}\text { Swallowing pathophysiology FEES findings } \\
\text { according to Desuter }\end{array}$ & $\begin{array}{l}\text { Swallowing pathophysiology FEES findings according to } \\
\text { Warnecke et al }\end{array}$ \\
\hline $\begin{array}{l}\text { Posterior oral incontinence } \\
\text { Delayed pharyngeal phase } \\
\text { Afferent }\end{array}$ & $\begin{array}{l}\text { Premature spillage } \\
\text { Delayed swallow reflex }\end{array}$ \\
Efferent & \\
Mixed & \\
Propulsion deficit & Impaired pharyngeal bolus clearance \\
Tongue base & \\
Pharyngeal musculature & \\
Resistive issue & Impaired UES opening \\
Protective deficit & Combination of different impairment patterns \\
Velopharyngeal insufficiency & \\
Impaired vocal fold motility & \\
Impaired laryngeal anatomy & \\
Oropharyngeal dyspraxia & Combination with pharyngo-laryngeal movement disorders \\
& Combination with fatigable swallowing \\
\hline
\end{tabular}

tomography (CT), magnetic resonance imaging (MRI), VFSS, HRM, swallowing electromyography (sEMG), and scintigraphy are usually necessary to complete the diagnostic process, especially with regard to the diagnosis of the underlying disorder. For example, in case of unexplained severe hypopharyngeal residue a CT scan with contrast is usually the first-choice examination to exclude a hypopharyngeal or proximal esophageal carcinoma before a surgical endoscopic procedure for tissue biopsy is scheduled. VFSS is the 'other' gold-standard assessment tool for swallowing and provides complimentary information next to the FEES examination [2]. VFSS can provide additional information in case of oral bolus preparation and/or oral transport impairment which cannot be observed during a FEES examination. Moreover, VFSS is recommended if hypopharyngeal residue is observed during a FEES examination because underlying mechanisms of this pooling such as a UES dysfunction and esophageal motility disorders can be revealed. In selected cases, VFSS is necessary to assess the pharyngeal stripping wave, hyolaryngeal elevation, the timing of airway protection mechanisms (laryngeal vestibule closure, hyolaryngeal elevation, epiglottis tilt), and the hypopharyngeal space if osteophytes block the endoscopic view. HRM should be considered when pharyngeal constrictor muscle and UES impairment are expected as HRM provides unique information on intraluminal pharyngo-esophageal pressure gradients during swallowing $[132,133]$. sEMG can also provide additional information on the UES function based on the analysis of submental and cricopharyngeal muscle activation and timing [134] and can contribute to decision making about the indication for botulinum toxin injection in the UES [135].

\section{Future developments for FEES}

As mentioned above, technological innovations have contributed to the refinement, further development, and increased diagnostic accuracy of the FEES technique. Furthermore, magnification endoscopy, high-resolution endoscopy, and chromoscopy have contributed to an improved optical diagnosis including the detection of minor abnormalities of the surface mucosa [136]. In addition, spectral narrowband filters used in narrow-band imaging (NBI) can help to visualize minor abnormalities of the surface mucosa and altered vascular patterns that may be consistent with premalignant lesions [137]. Moreover, the application of spectral narrow-band filters improved the detection rate and precise localization of minor bolus residue enhancing the diagnostic accuracy of penetration and aspiration [18, 138]. The further development of low-cost video capturing systems including applications for the smartphone is ongoing, as is the use of Wi-Fi-based wireless FEES systems [139, 140].

More than 30 years after its first description, FEES is still 'work in progress' and new innovative achievements are expected for the future [64]. The most important need is to increase the number of FEES certified trained health professionals to serve the underserved, thus reducing the discrepancy between instrumental assessment demand and its availability. Hands-on FEES courses as the one developed in Japan by the Japanese Society of Dysphagia Rehabilitation (JSDR) [48] or in Europe by the ESSD [47], telemedicine between patients and health professionals, and the development of books and e-learning materials on FEES [141] need to be expanded and further improved. The state of the art of a FEES examination and the minimum standards of care in this context will vary by country and will depend on the 
setting of the medical care (e.g., acute hospital, rehabilitation unit, and community nursing home), the personal preferences of health professionals, and the resources available for FEES education and equipment. Given the need for a consensus worldwide, further collaboration between different stakeholders (health professionals, patients, policy makers, manufacturers) is a wish for the near future. Important points of attention for the future are the development and validation of FEES protocols and psychometrically sound FEES metrics for different pathophysiological conditions and varying clinical settings. In addition, research funding to study and achieve these major points of attention deserves a fair chance. Research grants usually tend to focus on treatment of the underlying disease itself than on the functional impairments or consequences of the disease such as OD or its treatment. Yet, it is important to note that the survival rate of many OD-causing diseases did not increase spectacularly in recent decades and patients in the short- or long-term die as a result of the disease (amyotrophic lateral sclerosis, head and neck cancer, etc.).

\section{Conclusion}

FEES is becoming increasingly popular and represents the first-choice OD assessment technique for both phoniatricians and otorhinolaryngologists because of their professional history with clinical experience in endoscopic assessment of the upper aerodigestive tract. The rising number of phoniatricians and otorhinolaryngologists performing FEES contributes to the early detection and treatment of OD in an aging European population. Nevertheless, a multidisciplinary approach together with other disciplines is crucial for the success of OD management. FEES provides complementary information on the swallowing function next to other instrumental assessment techniques such as VFSS, HRM, and sEMG. The combination of this information permits a more accurate description of the pathophysiological mechanisms of OD and a subsequent patient-tailored OD treatment plan. Several advancements of the FEES equipment, examination, and measurement protocols were made since its first description. Nevertheless, to date there is no consensus regarding the FEES examination protocol, metrics or measurement protocol to analyze FEES video recordings. However, the authors of this narrative review recommend the implementation of at least a standardized FEES examination and measurement protocol within each center to allow swallowing assessment which is able to detect changes in swallowing function over time in dysphagic patients.

Important points of attention for the future are an increase of the number of FEES certified trained health professionals, the development and validation of FEES protocols and metrics for different pathophysiological conditions and varying clinical settings.

Funding None.

Availability of data and material Not applicable.

Code availability Not applicable.

\section{Declarations}

Conflict of interest The authors declares that they have no conflict of interest.

\section{References}

1. Langmore S (1988) Fiberoptic endoscopic examination of swallowing safety: a new procedure. Dyphagia 2:216-219. https:// doi.org/10.1007/BF02414429

2. Langmore SE (2003) Evaluation of oropharyngeal dysphagia: which diagnostic tool is superior? Curr Opin Otolaryngol Head Neck Surg 11:485-489. https://doi.org/10.1097/00020840-20031 2000-00014

3. Weir N (2000) Otorhinolaryngology. Postgrad Med J 76:65-69. https://doi.org/10.1136/pmj.76.892.65

4. Am Zehnhoff-Dinnesen A, Wiskirska-Woznica B, Neumann K, Nawka T (2020) Phoniatrics I. Springer, Germany

5. Bolger WE, Kennedy DW (1992) Nasal endoscopy in the outpatient clinic. Otolaryngol Clin North Am 25:791-802

6. Yanagisawa E (1994) The use of video in ENT endoscopy: its value in teaching. Ear Nose Throat J 73:754-763

7. Rosen CA, Murry T (2000) Diagnostic laryngeal endoscopy. Otolaryngol Clin North Am 33:751-757. https://doi.org/10.1016/ s0030-6665(05)70241-3

8. Schindler A, Spadola Bisetti M, Favero E, Musto R, Ottaviani F, Schindler O (2005) Role of videoendoscopy in phoniatrics: data from three years of daily practice. Acta Otorhinolaryngol Ital 25:43-49

9. Garcia M (1855) Observations on the human voice. Proc R Soc London 7:397-410

10. Wendler J (1967) Zur Bedeutung der Stimmstarke bei der stroboskopischen Untersuchung. Folia Phoniatr 19:73-75

11. Zeitels SM (1999) Universal modular glottiscope system: the evolution of a century of design and technique for direct laryngoscopy. Ann Otol Rhinol Laryngol Suppl 179:2-24. https://doi. org/10.1177/00034894991080s901

12. Davidson TM, Bone RC, Nahum AM (1974) Flexible fiberoptic laryngobronchoscopy. Laryngoscope 84:1876-1882. https://doi. org/10.1002/lary.5540841102

13. Wei WI, Lau WF, Lam KH, Hui Y (1987) The role of the fibreoptic bronchoscope in otorhinolaryngological practice. J Laryngol Otol 101:1263-1270. https://doi.org/10.1017/s00222151001036 39

14. Ikeda S, Yanai N, Ishikawa S (1968) Flexible bronchofiberscope. Keio J Med 17:1-16. https://doi.org/10.2302/kjm.17.1

15. Bastian RW (1991) Videoendoscopic evaluation of patients with dysphagia: an adjunct to the modified barium swallow. Otolaryngol Head Neck Surg 104:339-350. https://doi.org/10.1177/01945 9989110400309

16. Aviv JE, Kim T, Sacco RL, Kaplan S, Goodhart K, Diamond B, Close LG (1998) FEESST: a new bedside endoscopic test of the 
motor and sensory components of swallowing. Ann Otol Rhinol Laryngol 107:378-387. https://doi.org/10.1177/0003489498 10700503

17. Schröter-Morasch H, Bartolome G, Troppmann N, Ziegler W (1999) Values and limitations of pharyngolaryngoscopy (transnasal, transoral) in patients with dysphagia. Folia Phoniatr Logop 51:172-182. https://doi.org/10.1159/000021495

18. Nienstedt JC, Müller F, Nießen A, Fleischer S, Koseki JC, Flügel T, Pflug C (2017) Narrow band imaging enhances the detection rate of penetration and aspiration in FEES. Dysphagia 32:443448. https://doi.org/10.1007/s00455-017-9784-4

19. Mazerolles M, Woisard V (2003) Evaluation and treatment of swallowing disorders after tracheal intubation and tracheotomy. Rev Laryngol Otol Rhinol (Bord) 124(5):325-330

20. Hartnick CJ, Hartley BE, Miller C, Willging JP (2000) Pediatric fiberoptic endoscopic evaluation of swallowing. Ann Otol Rhinol Laryngol 109:996-999. https://doi.org/10.1177/0003489400 10901102

21. Verdonschot RJCG, Baijens LWJ, Vanbelle S, Florie M, Dijkman R, Leeters IPM, Bernd Kremer B, Leue C (2019) Medically unexplained oropharyngeal dysphagia at the university hospital ENT outpatient clinic for dysphagia: a cross-sectional cohort study. Dysphagia 34:43-51. https://doi.org/10.1007/ s00455-018-9912-9

22. Arens C, Herrmann IF, Rohrbach S, Schwemmle C, Nawka T (2015) Position paper of the German society of oto-rhino-laryngology, head and neck surgery and the German society of phoniatrics and pediatric audiology - current state of clinical and endoscopic diagnostics, evaluation, and therapy of swallowing disorders in children and adults. Laryngorhinootologie 94(Suppl 1):S306-S354. https://doi.org/10.1055/s-0035-1545298

23. Farneti D, Schindler A, Fattori B, Ruoppolo G, Simonelli M, Coscarelli S, Travalca Cupillo B, Spadola Bisetti M, Nacci A, Genovese E, Barillari U, Schindler O (2018) The role of the audiologist-phoniatrician in performing the dynamic endoscopic study of swallowing. Position statement of the Italian study group on dysphagia (GISD). Hear Balanc Comm 16:280-283. https:// doi.org/10.1080/21695717.2017.1395168

24. Starmer HM, Ayoub N, Byward C, Kizner J, Le Q, Hara W, Holsinger FC (2017) The impact of developing a speech and swallow rehab program: Improving patient satisfaction and multidisciplinary care. Laryngoscope 127:2578-2581. https://doi.org/10. 1002/lary.26695

25. Pflug C, Flügel T, Nienstedt JC (2018) Developments in dysphagia diagnostics: presentation of an interdisciplinary concept. HNO 66:506-514. https://doi.org/10.1007/s00106-017-0433-x

26. Aviv JE, Kaplan ST, Thomson JE, Spitzer J, Diamond B, Close LG (2000) The safety of flexible endoscopic evaluation of swallowing with sensory testing (FEESST): an analysis of 500 consecutive evaluations. Dysphagia 15:39-44. https://doi.org/10. 1007/s004559910008

27. Aviv JE, Murry T, Zschommler A, Cohen M, Gartner C (2005) Flexible endoscopic evaluation of swallowing with sensory testing: patient characteristics and analysis of safety in 1340 consecutive examinations. Ann Otol Rhinol Laryngol 114:173-176. https://doi.org/10.1177/000348940511400301

28. Warnecke T, Teismann I, Oelenberg S, Hamacher C, Ringelstein EB, Schabitz WR, Dziewas R (2009) The safety of fiberoptic endoscopic evaluation of swallowing in acute stroke patients. Stroke 40:482-486. https://doi.org/10.1161/STROKEAHA.108. 520775

29. Nacci A, Matteucci J, Romeo SO, Santopadre S, Cavaliere MD, Barillari MR, Berrettini S, Fattori B (2016) Complications with Fiberoptic endoscopic evaluation of swallowing in 2,820 examinations. Folia Phoniatr Logop 68:37-45. https://doi.org/10.1159/ 000446985
30. Dziewas R, auf dem Brinke M, Birkmann U et al (2019) Safety and clinical impact of FEES-results of the FEESregistry. Neurol Res Pract 1:16. https://doi.org/10.1186/ s42466-019-0021-5

31. O'Dea MB, Langmore SE, Krisciunas GP, Walsh M, Zanchetti LL, Scheel R, McNally E, Kaneoka AS, Guarino AJ, Butler SG (2015) Effect of lidocaine on swallowing during FEES in patients with dysphagia. Ann Otol Rhinol Laryngol 124:537-544. https:// doi.org/10.1177/0003489415570935

32. Hafner G, Neuhuber A, Hirtenfelder S, Schmedler B, Eckel HE (2008) Fiberoptic endoscopic evaluation of swallowing in intensive care unit patients. Eur Arch Otorhinolaryngol 265:441-446. https://doi.org/10.1007/s00405-007-0507-6

33. Reynolds J, Carroll S, Sturdivant C (2016) Fiberoptic endoscopic evaluation of swallowing: a multidisciplinary alternative for assessment of infants with dysphagia in the neonatal intensive care unit. Adv Neonatal Care 16:37-43. https://doi.org/10.1097/ ANC.0000000000000245

34. Imaizumi M, Suzuki T, Ikeda M, Matsuzuka T, Goto A, Omori K (2020) Implementing a flexible endoscopic evaluation of swallowing at elderly care facilities to reveal characteristics of elderly subjects who screened positive for a swallowing disorder. Auris Nasus Larynx 47:602-608. https://doi.org/10.1016/j.anl.2020. 02.004

35. Frajkova Z, Tedla M, Tedlova E, Suchankova M, Geneid A (2020) Postintubation dysphagia during COVID-19 outbreakcontemporary review. Dysphagia 35:549-557. https://doi.org/ 10.1007/s00455-020-10139-6

36. Collins WO (2009) A review of reprocessing techniques of flexible nasopharyngoscopes. Otolaryngol Head Neck Surg 141:307-310. https://doi.org/10.1016/j.otohns.2009.05.027

37. Oh HJ, Kim JS (2015) Clinical practice guidelines for endoscope reprocessing. Clin Endosc 48:364-368. https://doi.org/10.5946/ ce.2015.48.5.364

38. Deutschmann MW, McDonough A, Dort JC, Dort E, Nakoneshny S, Matthews TW (2013) Fiber-optic endoscopic evaluation of swallowing (FEES): predictor of swallowing-related complications in the head and neck cancer population. Head Neck 35:974979. https://doi.org/10.1002/hed.23066

39. Böckler R (2016) FEES in infants with swallowing disorders-a feasible procedure? Laryngorhinootologie 95:192-196. https:// doi.org/10.1055/s-0035-1555886

40. Braun T, Juenemann M, Viard M, Meyer M, Fuest S, Reuter I, Kaps M, Prosiegel M, Tanislav C (2018) What is the value of fibre-endoscopic evaluation of swallowing (FEES) in neurological patients? A cross-sectional hospital-based registry study. BMJ Open 8:e019016. https://doi.org/10.1136/bmjopen-2017-019016

41. Braun T, Juenemann M, Viard M, Meyer M, Reuter I, Prosiegel M, Kaps M, Tanislav C (2019) Adjustment of oral diet based on flexible endoscopic evaluation of swallowing (FEES) in acute stroke patients: a cross-sectional hospital-based registry study. BMC Neurol 19:282. https://doi.org/10.1186/s12883-019-1499-8

42. Braun T, Juenemann M, Viard M, Meyer M, Reuter I, Mausbach S, Doerr JM, Schirotzek I, Prosiegel M, Schramm P, Kaps M, Tanislav C (2020) Flexible endoscopic evaluation of swallowing (FEES) to determine neurological intensive care patients' oral diet. Int J Speech Lang Pathol 3:1-9. https://doi.org/10.1080/ 17549507.2020.1744727

43. ASHA Special Interest Division 13: swallowing and swallowing disorders (dysphagia) (1997) Graduate curriculum on swallowing and swallowing disorders. Asha Desk Reference 3:248a-248n

44. American Speech-Language-Hearing Association (2002) Roles of speech-language pathologists in swallowing and feeding disorders: technical report. Asha Desk Reference 3:181-199

45. American Speech-Language-Hearing Association (2002) Knowledge and skills for speech-language pathologists 
performing endoscopic assessment of swallowing functions. ASHA Suppl 22:107-112

46. American Speech-Language-Hearing Association (2002) The role of the speech-language pathologist in the performance and interpretation of endoscopic evaluation of swallowing: technical report. ASHA Suppl 2002(25):1-5

47. Dziewas R, Baijens L, Schindler A, Verin E, Michou E, Clave P (2017) European society for swallowing disorders FEES accreditation program for neurogenic and geriatric oropharyngeal dysphagia. Dysphagia 32:725-733. https://doi.org/10. 1007/s00455-017-9828-9

48. Yoshida M, Kagaya H, Kamakura Y, Miura Y, Saitoh E, Okawa Y, Sanada H (2020) Safety and the effectiveness of a new education program for nurses to assess swallowing function using fiberoptic endoscopic evaluation of swallowing (FEES). Jpn J Nurs Sci 17:e12313. https://doi.org/10.1111/jjns.12313

49. Pilz W, Vanbelle S, Kremer B, van Hooren MR, van Becelaere T, Roodenburg N, Baijens LW (2016) Observers' agreement on measurement in fiberoptic endoscopic evaluation of swallowing. Dysphagia 31:180-187. https://doi.org/10.1007/ s00455-015-9673-7

50. Périé S, Dernis HP, Monceaux G, Angelard B, St Guily JL (1999) The "sign of the rising tide" during swallowing fiberoscopy: a specific manifestation of Zenker's diverticulum. Ann Otol Rhinol Laryngol 108:296-299. https://doi.org/10.1177/ 000348949910800314

51. Gandor F, Vogel A, Claus I, Ahring S, Gruber D, Heinze HJ, Dziewas R, Ebersbach G, Warnecke T (2020) Laryngeal movement disorders in multiple system atrophy: a diagnostic biomarker? Mov Disord. https://doi.org/10.1002/mds.28220 (Online ahead of print)

52. Cortelli P, Calandra-Buonaura G, Benarroch EE, Giannini G, Iranzo A, Low PA, Martinelli P, Provini F, Quinn N, Tolosa E, Wenning GK, Abbruzzese G, Bower P, Alfonsi E, Ghorayeb I, Ozawa T, Pacchetti C, Pozzi NG, Vicini C, Antonini A, Bhatia KP, Bonavita J, Kaufmann H, Pellecchia MT, Pizzorni N, Schindler A, Tison F, Vignatelli L, Meissner WG (2019) Stridor in multiple system atrophy: consensus statement on diagnosis, prognosis, and treatment. Neurology 93:630-639. https://doi.org/10.1212/WNL.0000000000008208

53. Argolo N, Sampaio M, Pinho P, Melo A, Nobrega AC (2015) Swallowing disorders in Parkinson's disease: impact of lingual pumping. Int J Lang Commun Disord 50:659-664. https://doi. org/10.1111/1460-6984.12158

54. Warnecke T, Teismann I, Zimmermann J, Oelenberg S, Ringelstein EB, Dziewas R (2008) Fiberoptic endoscopic evaluation of swallowing with simultaneous Tensilon application in diagnosis and therapy of myasthenia gravis. J Neurol 255:224-230. https://doi.org/10.1007/s00415-008-0664-6

55. Warnecke T, Suttrup I, Schröder JB, Osada N, Oelenberg S, Hamacher C, Suntrup S, Dziewas R (2016) Levodopa responsiveness of dysphagia in advanced Parkinson's disease and reliability testing of the FEES-Levodopa-test. Parkinsonism Relat Disord 28:100-106. https://doi.org/10.1016/j.parkreldis.2016. 04.034

56. Chen JR, Mirghani H, Jafari A, de Crouy CO, Périé S, Lacau St Guily J (2013) Role of the "rising tide sign" in the diagnosis and assessment of the results of surgery for Zenker's diverticulum. Eur Ann Otorhinolaryngol Head Neck Dis 130:309-311. https:// doi.org/10.1016/j.anorl.2011.11.005

57. Swan K, Cordier R, Brown T, Speyer R (2019) Pyschometric properties of visuoperceptual measures of videofluoroscopic and fibre-endoscopic evaluations of swallowing: a systematic review. Dysphagia 34:2-33. https://doi.org/10.1007/s00455-018-9918-3

58. Leder SB (1998) Serial fiberoptic endoscopic swallowing evaluations in the management of patients with dysphagia. Arch Phys
Med Rehabil 79:1264-1269. https://doi.org/10.1016/s00039993(98)90273-8

59. Cvejic L, Harding R, Churchward T, Turton A, Finlay P, Massey D, Bardin PG, Guy P (2011) Laryngeal penetration and aspiration in individuals with stable COPD. Respirology 16:269-275. https://doi.org/10.1111/j.1440-1843.2010.01875.x

60. Hammond CAS, Goldstein LB (2006) Cough and aspiration of food and liquids due to oral-pharyngeal dysphagia: ACCP evidence-based clinical practice guidelines. Chest 129(1 Suppl):154S-168S. https://doi.org/10.1378/chest.129.1_suppl. $154 \mathrm{~S}$

61. Dziewas R, Warnecke T, Olenberg S, Teismann I, Zimmermann J, Kramer C, Ritter M, Ringelstein EB, Schabitz WR (2008) Towards a basic endoscopic assessment of swallowing in acute stroke-development and evaluation of a simple dysphagia score. Cerebrovasc Dis 26:41-47. https://doi.org/10.1159/000135652

62. Warnecke T, Suntrup S, Teismann IK, Hamacher C, Oelenberg S, Dziewas R (2013) Standardized endoscopic swallowing evaluation for tracheostomy decannulation in critically ill neurologic patients. Crit Care Med 41:1728-1732. https://doi.org/10.1097/ CCM.0b013e31828a4626

63. Pilz W, Baijens LW, Passos VL, Verdonschot R, Wesseling F, Roodenburg N, Faber CG, Kremer B (2014) Swallowing assessment in myotonic dystrophy type 1 using fiberoptic endoscopic evaluation of swallowing (FEES). Neuromuscul Disord 24:1054 1062. https://doi.org/10.1016/j.nmd.2014.06.002

64. Langmore SE (2017) History of fiberoptic endoscopic evaluation of swallowing for evaluation and management of pharyngeal dysphagia: changes over the years. Dysphagia 32:27-38

65. Langmore S (2001) Endoscopic evaluation and treatment of swallowing disorders, 1st edn. Thieme, New York

66. Denk DM, Kaider A (1997) Videoendoscopic biofeedback: a simple method to improve the efficacy of swallowing rehabilitation of patients after head and neck surgery. ORL J Otorhinolaryngol Relat Spec 59:100-105. https://doi.org/10.1159/ 000276918

67. Leder SB, Novella S, Patwa H (2004) Use of fiberoptic evaluation of swallowing (FEES) in patients with amyotrophic lateral sclerosis. Dysphagia 19:177-181. https://doi.org/10.1007/ s00455-004-0009-2

68. Desuter G (2019) Video-endoscopy by screenplays. In: Desuter $\mathrm{G}$ (ed) Oropharyngeal dysphagia. Springer Nature, Cham, Swaitzerland, pp 9-41

69. Colquhoun-Flannery W, Davis A, Carruth JA (2000) Improving the endoscopic view of the hypopharynx with anterior neck traction during the trumpet manoeuvre. J Laryngol Otol 114:283284. https://doi.org/10.1258/0022215001905562

70. Donzelli J, Brady S, Wesling M, Craney M (2001) Simultaneous modified Evans blue dye procedure and video nasal endoscopic evaluation of the swallow. Laryngoscope 111:1746-1750. https:// doi.org/10.1097/00005537-200110000-00015

71. Ricci Maccarini A, Stacchini M, Salsi D, Pieri F, Magnani M, Casolino D (2007) Trans-tracheostomic endoscopy of the larynx in the evaluation of dysphagia. Acta Otorhinolaryngol Ital 27:290-293

72. Bastian RW (1993) The videoendoscopic swallowing study: an alternative and partner to the videofluoroscopic swallowing study. Dysphagia 8:359-367. https://doi.org/10.1007/BF013 21780

73. Leder SB, Acton LM, Lisitano HL, Murray JT (2005) Fiberoptic endoscopic evaluation of swallowing (FEES) with and without blue-dyed food. Dysphagia 20:157-162. https://doi.org/10.1007/ s00455-005-0009-x

74. Marvin S, Gustafson S, Thibeault S (2016) Detecting aspiration and penetration using FEES with and without food dye. Dysphagia 31:498-504. https://doi.org/10.1007/s00455-016-9703-0 
75. Tariq B, Simon SR, Pilz W, Maxim A, Kremer B, Baijens LWJ (2021) Evaluating safety of methylene blue during swallowing assessment: a systematic review. Eur Arch OtoRhino Laryngol 278:3155-3169. https://doi.org/10.1007/s00405-020-06509-3

76. Curtis J, Perry S, Troche MS (2019) Detection of airway invasion during flexible endoscopic evaluations of swallowing: comparing barium, blue dye, and green dye. Am J Speech Lang Pathol 28:515-520. https://doi.org/10.1044/2018_ AJSLP-18-0119

77. Baijens LW, Speyer R, Pilz W, Roodenburg N (2014) FEES protocol derived estimates of sensitivity: aspiration in dysphagic patients. Dysphagia 29:583-590. https://doi.org/10.1007/ s00455-014-9549-2

78. Martin-Harris B, Humphries K, Garand KL (2017) The modified barium swallow impairment profile (MBSImP $\mathrm{PC}^{\mathrm{TC}}$ () - - innovation, dissemination and implementation. Pers ASHA Special Int Group 2:129-138. https://doi.org/10.1044/persp2.SIG13.129

79. Pisegna JM, Murray J (2018) Clinical application of flexible endoscopic evaluation of swallowing in stroke. Semin Speech Lang 39:3-14. https://doi.org/10.1055/s-0037-1608855

80. McCabe D, Ashford J, Wheeler-Hegland K, Frymark T, Mullen R, Musson N, Hammond CS, Schooling T (2009) Evidencebased systematic review: Oropharyngeal dysphagia behavioral treatments. Part IV - impact of dysphagia treatment on individuals' postcancer treatments. J Rehabil Res Dev 46:205-214

81. Ashford J, McCabe D, Wheeler-Hegland K, Frymark T, Mullen R, Musson N, Schooling T, Hammond CS (2009) Evidencebased systematic review: oropharyngeal dysphagia behavioral treatments. Part III-impact of dysphagia treatments on populations with neurological disorders. J Rehabil Res Dev 46:195-204

82. Wheeler-Hegland K, Ashford J, Frymark T, McCabe D, Mullen R, Musson N, Hammond CS, Schooling T (2009) Evidencebased systematic review: oropharyngeal dysphagia behavioral treatments. Part II-impact of dysphagia treatment on normal swallow function. J Rehabil Res Dev 46:185-194

83. European Society for Swallowing Disorders (2013) ESSD position statements. Dysphagia 28:280-282

84. Willging JP (1995) Endoscopic evaluation of swallowing in children. Int J Pediatr Otorhinolaryngol 32 Suppl:S107-S108. https://doi.org/10.1016/0165-5876(94)01174-v

85. Miller CK, Willging JP (2020) Fiberoptic endoscopic evaluation of swallowing in infants and children: protocol, safety, and clinical efficacy: 25 years of experience. Ann Otol Rhinol Laryngol 129:469-481. https://doi.org/10.1177/0003489419893720

86. Miller CK, Schroeder JW Jr, Langmore S (2020) Fiberoptic endoscopic evaluation of swallowing across the age spectrum. Am J Speech Lang Pathol 29(2S):967-978. https://doi.org/10.1044/ 2019_AJSLP-19-00072

87. Willette S, Molinaro LH, Thompson DM, Schroeder JW Jr (2016) Fiberoptic examination of swallowing in the breastfeeding infant. Laryngoscope 126:1681-1686. https://doi.org/10.1002/ lary. 25641

88. Rosenbek JC, Robbins JA, Roecker EB, Coyle JL, Wood JL (1996) A penetration-aspiration scale. Dysphagia 11:93-98. https://doi.org/10.1007/BF00417897

89. Rodriguez KH, Roth CR, Rees CJ, Belafsky PC (2007) Reliability of the pharyngeal squeeze maneuver. Ann Otol Rhinol Laryngol 116:399-401. https://doi.org/10.1177/0003489407 11600601

90. Fuller SC, Leonard R, Aminpour S, Belafsky PC (2009) Validation of the pharyngeal squeeze maneuver. Otolaryngol Head Neck Surg 140:391-394. https://doi.org/10.1016/j.otohns.2008. 12.015

91. Aviv JE, Spitzer J, Cohen M, Ma G, Belafsky P, Close LG (2002) Laryngeal adductor reflex and pharyngeal squeeze as predictors of laryngeal penetration and aspiration. Laryngoscope
112:338-341. https://doi.org/10.1097/00005537-20020 2000-00025

92. Borders JC, O’'Dea MB, McNally E, Norberg E, Kitila M, Walsh M, Liu R, Pisegna JM (2020) Inter- and intra-rater reliability of laryngeal sensation testing with the touch method during flexible endoscopic evaluations of swallowing. Ann Otol Rhinol Laryngol 129:565-571. https://doi.org/10.1177/0003489419901145

93. Kaneoka A, Pisegna JM, Krisciunas GP, Nito T, LaValley MP, Stepp CE, Langmore SE (2017) Variability of the pressure measurements exerted by the tip of laryngoscope during laryngeal sensory testing: a clinical demonstration. Am J Speech Lang Pathol 26:729-736. https://doi.org/10.1044/2017_AJSLP-16-0006

94. Kaneoka A, Krisciunas GP, Walsh K, Raade AS, Langmore SE (2015) A comparison of 2 methods of endoscopic laryngeal sensory testing: a preliminary study. Ann Otol Rhinol Laryngol 124:187-193. https://doi.org/10.1177/0003489414550241

95. Kaneoka A, Pisegna JM, Inokuchi H, Ueha R, Goto T, Nito T, Stepp CE, LaValley MP, Haga N, Langmore SE (2018) Relationship between laryngeal sensory deficits, aspiration, and pneumonia in patients with dysphagia. Dysphagia 33:192-199. https:// doi.org/10.1007/s00455-017-9845-8

96. Langmore SE, Olney RK, Lomen-Hoerth C, Miller BL (2007) Dysphagia in patients with frontotemporal lobar dementia. Arch Neurol 64:58-62. https://doi.org/10.1001/archneur.64.1.58

97. Zancan M, Luchesi KF, Mituuti CT, Furkim AM (2017) Onset locations of the pharyngeal phase of swallowing: meta-analysis. Codas 29:e20160067. https://doi.org/10.1590/2317-1782/20172 016067

98. Young JL, Macrae P, Anderson C, Taylor-Kamara I, Humbert IA (2015) The sequence of swallowing events during the chin-down posture. Am J Speech Lang Pathol 24:659-670. https://doi.org/ 10.1044/2015_AJSLP-15-0004

99. Calvo I, Sunday KL, Macrae P, Humbert IA (2017) Effects of chin-up posture on the sequence of swallowing events. Head Neck 39:947-959. https://doi.org/10.1002/hed.24713

100. Nagy A, Leigh C, Hori SF, Molfenter SM, Shariff T, Steele CM (2013) Timing differences between cued and noncued swallows in healthy young adults. Dysphagia 28:428-434. https://doi.org/ 10.1007/s00455-013-9456-y

101. Dua KS, Ren J, Bardan E, Xie P, Shaker R (1997) Coordination of deglutitive glottal function and pharyngeal bolus transit during normal eating. Gastroenterology 112:73-83. https://doi.org/10. 1016/s0016-5085(97)70221-x

102. Clavé P, de Kraa M, Arreola V, Girvent M, Farré R, Palomera E, Serra-Prat M (2006) The effect of bolus viscosity on swallowing function in neurogenic dysphagia. Aliment Pharmacol Ther 24:1385-1394. https://doi.org/10.1111/j.1365-2036.2006. 03118.x

103. Steele CM, Alsanei WA, Ayanikalath S, Barbon CE, Chen J, Cichero JA, Coutts K, Dantas RO, Duivestein J, Giosa L, Hanson B, Lam P, Lecko C, Leigh C, Nagy A, Namasivayam AM, Nascimento WV, Odendaal I, Smith CH, Wang H (2015) The influence of food texture and liquid consistency modification on swallowing physiology and function: a systematic review. Dysphagia 30:2-26. https://doi.org/10.1007/s00455-014-9578-x

104. Humbert IA, Sunday KL, Karagiorgos E, Vose AK, Gould F, Greene L, Azola A, Tolar A, Rivet A (2018) Swallowing kinematic differences across frozen, mixed, and ultrathin liquid boluses in healthy adults: age, sex, and normal variability. $\mathbf{J}$ Speech Lang Hear Res 61:1544-1559. https://doi.org/10.1044/ 2018_JSLHR-S-17-0417

105. Logemann JA, Pauloski BR, Colangelo L, Lazarus C, Fujiu M, Kahrilas PJ (1995) Effects of a sour bolus on oropharyngeal swallowing measures in patients with neurogenic dysphagia. J Speech Hear Res 38:556-563. https://doi.org/10.1044/jshr.3803.556 
106. Bülow M, Olsson R, Ekberg O (2003) Videoradiographic analysis of how carbonated thin liquids and thickened liquids affect the physiology of swallowing in subjects with aspiration on thin liquids. Acta Radiol 44:366-372. https://doi.org/10.1034/j.16000455.2003.00100.x

107. Shin S, Shutoh N, Tonai M, Ogata N (2016) The effect of capsaicin-containing food on the swallowing response. Dysphagia 31:146-153. https://doi.org/10.1007/s00455-015-9668-4

108. Steele CM, Peladeau-Pigeon M, Barrett E, Wolkin TS (2020) The risk of penetration-aspiration related to residue in the pharynx. Am J Speech Lang Pathol 29:1608-1617. https://doi.org/ 10.1044/2020_AJSLP-20-00042

109. Colodny $\mathrm{N}$ (2002) Interjudge and intrajudge reliabilities in fiberoptic endoscopic evaluation of swallowing (fees) using the penetration-aspiration scale: a replication study. Dysphagia 17:308-315. https://doi.org/10.1007/s00455-002-0073-4

110. Butler SG, Markley L, Sanders B, Stuart A (2015) Reliability of the penetration aspiration scale with flexible endoscopic evaluation of swallowing. Ann Otol Rhinol Laryngol 124:480-483. https://doi.org/10.1177/0003489414566267

111. Hey C, Pluschinski P, Pajunk R, Almahameed A, Girth L, Sader R, Stöver T, Zaretsky Y (2015) Penetration-aspiration: is their detection in FEES ${ }^{\circledR}$ reliable without video recording? Dysphagia 30:418-422. https://doi.org/10.1007/s00455-015-9616-3

112. Pizzorni N, Crosetti E, Santambrogio E, de Cillis G, Bertolin A, Rizzotto G, Fantini M, Succo G, Schindler A (2020) The penetration-aspiration scale: adaptation to open partial laryngectomy and reliability analysis. Dysphagia 35:261-271. https://doi. org/10.1007/s00455-019-10025-w

113. Schindler A, Pizzorni N, Sassone J, Nanetti L, Castaldo A, Poletti B, Solca F, Pirola F, Lazzari L, Stramba-Badiale M, Rossi A, Silani V, Mariotti C, Ciammola A (2020) Fiberoptic endoscopic evaluation of swallowing in early-to-advanced stage Huntington's disease. Sci Rep 10:15242. https://doi.org/10.1038/ s41598-020-72250-w

114. Schindler A, Mozzanica F, Sonzini G, Plebani D, Urbani E, Pecis M, Montano N (2014) Oropharyngeal dysphagia in patients with obstructive sleep apnea syndrome. Dysphagia 29:44-51. https:// doi.org/10.1007/s00455-013-9474-9

115. Pizzorni N, Ginocchio D, Bianchi F, Feroldi S, Vedrodyova M, Mora G, Schindler A (2020) Association between maximum tongue pressure and swallowing safety and efficacy in amyotrophic lateral sclerosis. Neurogastroenterol Motil 32:e13859. https://doi.org/10.1111/nmo.13859

116. Farneti D (2008) Pooling score: an endoscopic model for evaluating severity of dysphagia. Acta Otorhinolaryngol Ital 28:135-140

117. Kaneoka AS, Langmore SE, Krisciunas GP, Field K, Scheel R, McNally E, Walsh MJ, O’Dea MB, Cabral H (2013) The Boston residue and clearance scale: preliminary reliability and validity testing. Folia Phoniatr Logop 65:312-317. https://doi.org/10. 1159/000365006

118. Neubauer PD, Rademaker AW, Leder SB (2015) The yale pharyngeal residue severity rating scale: an anatomically defined and image-based tool. Dysphagia 30:521-528. https://doi.org/ 10.1007/s00455-015-9631-4

119. Neubauer PD, Hersey DP, Leder SB (2016) Pharyngeal residue severity rating scales based on fiberoptic endoscopic evaluation of swallowing: a systematic review. Dysphagia 31:352-359. https://doi.org/10.1007/s00455-015-9682-6

120. Murray J, Langmore SE, Ginsberg S, Dostie A (1996) The significance of accumulated oropharyngeal secretions and swallowing frequency in predicting aspiration. Dysphagia 11:99-103. https://doi.org/10.1007/BF00417898

121. Miles A, Hunting A (2019) Development, intra- and inter-rater reliability of the New Zealand Secretion Scale (NZSS). Int J
Speech Lang Pathol 21:377-384. https://doi.org/10.1080/17549 507.2018.1458901

122. Miles A, Hunting A, McFarlane M, Caddy D, Scott S (2018) Predictive value of the New Zealand Secretion Scale (NZSS) for Pneumonia. Dysphagia 33:115-122. https://doi.org/10. 1007/s00455-017-9841-z

123. Pisegna JM, Borders JC, Kaneoka A, Coster WJ, Leonard R, Langmore SE (2018) Reliability of untrained and experienced raters on FEES: rating overall residue is a simple task. Dysphagia 33:645-654. https://doi.org/10.1007/s00455-018-9883-x

124. Pisegna JM, Kaneoka A, Leonard R, Langmore SE (2018) Rethinking residue: determining the perceptual continuum of residue on FEES to enable better measurement. Dysphagia 33:100-108. https://doi.org/10.1007/s00455-017-9838-7

125. Williams RBH, Wallace KL, Ali GN, Cook IJ (2002) Biomechanics of failed deglutitive upper esophageal sphincter relaxation in neurogenic dysphagia. Am J Physiol Gastroeintest Liver Physiol 283:G16-G26. https://doi.org/10.1152/ajpgi.00189. 2001

126. Dejaeger E, Pelemans W, Ponette E, Joosten E (1997) Mechanisms involved in postdeglutition retention in the elderly. Dysphagia 12:63-67. https://doi.org/10.1007/PL00009520

127. Stokely SL, Peladeau-Pigeon M, Leigh C, Molfenter SM, Steele CM (2015) The relationship between pharyngeal constriction and post-swallow residue. Dysphagia 30:349-356. https://doi.org/10. 1007/s00455-015-9606-5

128. Logemann JA, Rademaker AW, Pauloski BR, Ohmae Y, Kahrilas PJ (1998) Normal swallowing physiology ans viewed by videofluoroscopy and videoendoscopy. Folia Phoniatr Logop 50:311-319. https://doi.org/10.1159/000021473

129. Mozzanica F, Lorusso R, Robotti C, Zambon T, Corti P, Pizzorni N, Vanderwegen J, Schindler A (2019) Effect of age, sex, bolus volume, and bolus consistency on whiteout duration in healthy subjects during FEES. Dysphagia 34:192-200. https://doi.org/ 10.1007/s00455-018-9961-0

130. Ertekin C, Aydoğdu I, Yüceyar N (1996) Piecemeal deglutitrion and dysphagia limit in normal subjects and in patients with swallowing disorders. J Neurol Neurosurg Psychiatry 61:491-496. https://doi.org/10.1136/jnnp.61.5.491

131. Warnecke T, Dziewas R, Langmore S (2020) Neurogenic dysphagia. Springer Nature, Cham, Swaitzerland

132. Omari T, Schar M (2018) High-resolution manometry: what about the pharynx? Curr Opin Otolaryngol Head Neck Surg 26:382-391. https://doi.org/10.1097/MOO.0000000000000491

133. Omari TI, Ciucci M, Gozdzikowska K, Hernández E, Hutcheson $\mathrm{K}$, Jones C, Maclean J, Nativ-Zeltzer N, Plowman E, Rogus-Pulia N, Rommel N, O'Rourke A (2020) High-resolution pharyngeal manometry and impedance: protocols and metrics-recommendations of a high-resolution pharyngeal manometry international working group. Dysphagia 35:281-295. https://doi.org/10.1007/ s00455-019-10023-y

134. Ertekin C (2002) Physiological and pathological aspects of oropharyngeal swallowing. Mov Disord 17(Suppl 2):S86-S89. https://doi.org/10.1002/mds. 10068

135. Alfonsi E, Restivo DA, Cosentino G, De Icco R, Bertino G, Schindler A, Todisco M, Fresia M, Cortese A, Prunetti P, Ramusino MC, Moglia A, Sandrini G, Tassorelli C (2017) Botulinum toxin is effective in the management of neurogenic dysphagia. Clinical-electrophysiological findings and tips on safety in different neurological disorders. Front Pharmacol 8:80. https:// doi.org/10.3389/fphar.2017.00080 (eCollection 2017)

136. Bruno MJ (2003) Magnification endoscopy, high resolution endoscopy, and chromoscopy; towards a better optical diagnosis. Gut 52(Suppl 4):iv7-11. https://doi.org/10.1136/gut.52. suppl_4.iv7 
137. Ansari UH, Wong E, Smith M, Singh N, Palme CE, Smith MC, Riffat F (2019) Validity of narrow band imaging in the detection of oral and oropharyngeal malignant lesions: asystematic review and meta-analysis. Head Neck 41:2430-2440. https://doi.org/10. 1002/hed.25724

138. Fleischer S, Pflug C, Hess M (2017) High-sensitivity FEES with NBI-illumination. Laryngoscope 127:1140-1142. https://doi.org/ 10.1002/lary.26328

139. Howes BW, Repanos C (2010) A low-cost, endoscopic, digital, still and video photography system for ENT clinics. J Laryngol Otol 124:543-544. https://doi.org/10.1017/S0022215109992428

140. Sakakura K, Tazawa M, Otani N, Takagi M, Morita M, Kurosaki M, Chiyoda T, Kanai Y, Endo A, Murata T, Shino M, Yokobori Y, Shirakura K, Wada N, Chikamatsu K (2017) Impact of a multidisciplinary round visit for the management of dysphagia utilizing a wi-fi-based wireless flexible endoscopic evaluation of swallowing. Ann Otol Rhinol Laryngol 126:47-53. https://doi. org/10.1177/0003489416674223

141. Brady SL, Rao N, Gibbons PJ, Williams L, Hakel M, pape T (2018) Face-to-face versus online training for the interpretation of findings in the fiberoptic endoscopic exam of the swallow procedure. Adv Med Educ Pract 9:433-441. https://doi.org/10. 2147/AMEP.S142947 (eCollection 2108)

Publisher's Note Springer Nature remains neutral with regard to jurisdictional claims in published maps and institutional affiliations. 